\title{
An Adaptive Approach to Active Fault-Tolerant Control
}

\author{
Ron J. Patton* and Supat Klinkhieo
}

Department of Engineering, University of Hull, UK

\begin{abstract}
In this Faults or process failures may drastically change system behaviour leading to performance degradation and instability. The reliability and fault-tolerance of a control system can be achieved through the design of either an active or passive Fault Tolerant Control (FTC) scheme. This paper proposes a new approach to fault compensation for FTC using fault estimation by which the faults acting in a dynamical system are estimated and compensated within an adaptive control scheme with required stability and performance robustness. The FTC scheme has an augmented state observer (ASO) in the control system, which has an intrinsic robustness in terms of the stability and performance of the estimation error. The design concepts are illustrated using the notion that the friction forces in a mechanical system can be estimated and compensated to give good control performance and stability. The example given is that of a non-linear inverted pendulum with Stribeck friction.
\end{abstract}

\section{INTRODUCTION}

The reliability, robustness and fault-tolerance of the control of uncertain systems are issues that have increasing importance as modern systems grow in complexity. In keeping with these developments robust methods for detecting and isolating faults have been developed that can robustly discriminate between the effects of uncertainty and the effects of faults acting within a system or on the actuators and/or sensors. This is the subject of robust fault detection and isolation (FDI) that has been based on the use of a variety of approaches, e.g. unknown input de-coupling, Ho [1], LPV [2], sliding mode estimation [3, 4] and non-linear geometric approaches [5].

Whilst robust FDI is concerned with the robust decision problem (detection, isolation and perhaps possible fault causes etc), the subject of FTC is concerned with the design and implementation of control schemes that are either active or passive in their method of reacting and compensating for faults $[6,7]$. During recent years there has been a substantial literature on the subject of FTC as reported in the review papers $[6,7]$ and book [8].

FTC can be motivated by different purposes depending on the application under consideration; for example; safety in flight control, efficiency and quality improvements in industrial processes, reliable of mechatronic systems, robotics, etc. The main design challenges are: (i) the number of possible faults acting on the system and their diagnosability, (ii) the system reconfigurability in terms of available redundancy etc, and (iii) the global stability of the system [8]. A fault can make the system deviate far from its normal operating conditions and can lead to severe change in system behaviour. Even bounded faults can cause the closed-loop system to deviate rapidly from its required operation and hence the

*Address correspondence to this author at the Department of Engineering, University of Hull, UK; Tel: 0044148241 5117; Fax: 0044148246 6664; E-mail: r.j.patton@hull.ac.uk

1874-4443/09 fault accommodation time is a critical parameter. The requirement for rapid reaction to faults can mean that an FDI procedure, if used, may slow down the accommodation process.

This paper is concerned with the active approach to FTC, following the classification of FTC systems as shown in Fig. (1), involving fault estimation, fault compensation and adaptive control. The controller is included within the structure of an ASO system in which the actuator faults are estimated via additional state components. The controller is designed using linear output feedback. However, the control system is adaptive as the on-line fault estimates are updated continuously and used to compensate the faults acting within the control channels. The compensation is achieved within the ASO estimation error system with the consequence that the control signal has a time-varying component, the adaptive part of the control.

The use of on-line compensation means that the fault isolation task of FDI is not required. The ideal residual generation problem of fault detection of FDI is replaced by robust fault estimation. It is of interest here to note that, as-

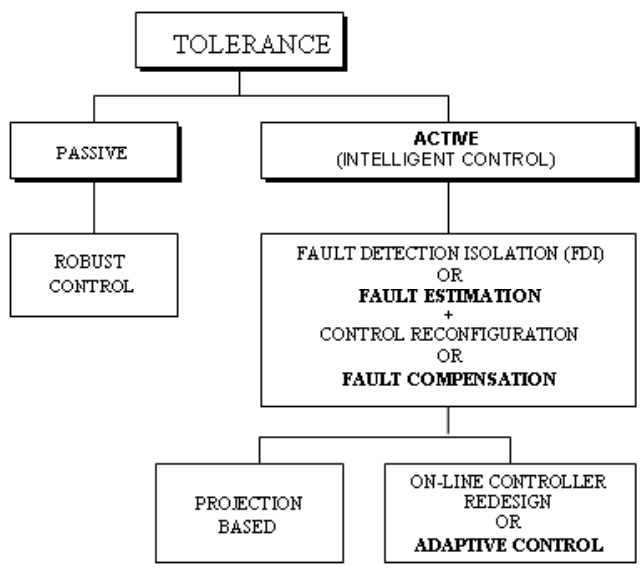

Fig. (1). Classification of FTC Systems (taken from [6]). 
suming a residual generator can be robust against modelling uncertainties, the residual signal or vector can be equivalent to a fault estimator under certain conditions which are described in the well known book by [9]. In this work we obviate the use of the residual generation process and turn directly to a robust fault estimation problem embedded within an adaptive control scheme.

In the linear system case our control scheme makes use of observer-based output feedback. However, in the non-linear case both fault(s) and modelling uncertainties (unknown inputs) is/are estimated and compensated using an augmented state space disturbance observer structure, the ASO, with additional states corresponding to estimates of both faults and uncertainties.

This paper focuses on the special case of actuator faults within an on-line fault estimation and compensation system using the ASO concept. The important principle is that the faults and modelling uncertainties both act to disturb the system dynamics. For clarity, we consider the case in which the fault compensation mechanism causes the closed-loop system to behave in an almost linear sense. However, further analysis and design shows that the approach easily handles cases of multiple faults and the co-existence of different types of faults (actuator, sensor or multiplicative faults) and unknown input signals (arising from the effect of modelling uncertainty in the estimation and control). The controller is adaptive as the fault/uncertainty estimation signal becomes a component in the state estimate feedback control, thereby cancelling bounded uncertainty effects due to either faults or unknown input signals acting on the observer state estimation error. The ASO includes a compensation gain matrix which must be designed using Lyapunov LMI-pole- placement approach, based on knowledge of the fault bounds. The paper discusses this theory via a theorem and corresponding proof.

The adaptive compensation FTC concept is illustrated by considering the friction force as a special type of input or actuator fault in a mechatronic system, the inverted pendulum. It is very reasonable to consider the friction as a fault in the system as it is an unwanted effect which causes the performance of the system to change. Patton, Putra and Klinkieo [10] tackled the friction force compensation problem using a sliding mode observer together with a sliding mode controller. The friction force could alternatively be described as having an uncertain effect in the system but we prefer here to consider the whole issue of friction compensation as an FTC problem. The friction (fault) estimation and compensation is handled in this paper using the ASO and the results demonstrate excellent performance of the adaptive controller in removing the effect of the friction force to yield very precise positioning control.

Extensive recent research has focused on detailed modeling of friction phenomena in order to use robust on-line friction compensation procedures [11-14]. However, the friction modeling problem remains a very difficult complex systems challenge and although complex modeling techniques are used no efficient method exists to ensure satisfactory robustness. It can easily be seen that the adaptive control approach used in this study obviates the need for explicit friction modeling. This offers significant advantages over well known model-based friction compensation methods in which detailed modeling of friction phenomena is essential and for which robustness with respect to friction characteristics is very difficult to achieve.

Section II outlines the proposed approach to fault estimation and compensation using the ASO concept. The controller has two components (a) state estimate feedback together with (b) the component arising from the fault compensation. Some analysis and proof of stability for this control system structure is given, showing that the controller fault compensation gain must lie in a defined interval derived via a Lyapunov stability condition, designed via the Matlab LMI toolbox. Section III describes the results arising from the inverted pendulum friction compensation problem. Section IV provides a concluding discussion with suggestions of further research.

\section{AUGMENTED STATE OBSERVER}

The idea of estimation of uncertain effects in an observer-based FDI scheme was discussed extensively in [9], in which the uncertain effects (modeling errors, unknown disturbances etc) are combined into unknown input signals. These authors also discussed the related problem of sensitizing the FDI observer estimation error to specific faults and de-sensitising the error dynamics to other faults - effectively a dual of the unknown input de-coupling problem. This problem is also discussed in [15]. As discussed in Section I, a side-step from this is to consider a problem of fault or unknown input estimation, using robust estimation techniques. The unknown input estimation problem was considered in [9, $16,17]$ using the ASO concept and this has motivated the current FTC study. The work by Patton and Chen did not make use of fault compensation within a control loop. Here we use the ASO concept in an FTC scheme, as outlined in Section I.

\section{ACTUATOR FAULT ESTIMATION}

Considering the state space representation of faulty system

$\dot{x}=A x+B u+F_{a} f_{a}$
$y=C x$

where $x \in \Re^{n}$ is the state vector, $y \in \Re^{p}$ the output

observation vector, $u \in \Re^{m}$ the input vector and $A, B$ and

$C$ are known matrices of appropriate dimensions. $F_{a}$ is the

fault distribution matrix for the actuator fault $f_{a} \in \mathfrak{R}^{m}$

corresponding to the $i^{t h}$ column of $B$ (in the case of the $i^{t h}$

actuator fault). This idea is considered even further here in the context of "an observer-based adaptive controller" of the form: 
$u=K_{x} \hat{x}+K_{f} \hat{f}_{a}$

A suitable analysis and design is required to stabilize the faulty system (1) around the origin in the presence of unwanted but bounded actuator fault signals. $K_{x} \in R^{m \times n}$ and $K_{f} \in R^{m \times m}$ are the controller and actuator fault compensation gains, respectively. The vectors $\hat{x}$ and $\hat{f}_{a}$ are the state and actuator fault estimation signals, respectively obtained from the ASO with dynamics derived as follows:

Substitute (2) into (1), giving

$\dot{x}=A x+B K_{x} \hat{x}+B K_{f} \hat{f}_{a}+F_{a} f_{a}$

$\dot{\hat{x}}=\left(A+B K_{x}\right) \hat{x}+L_{x}(y-C \hat{x})$

$\hat{f}_{a}=L_{f}(y-C \hat{x})$

Eqs. (4) and (5), can be re-arranged as:

$$
\begin{aligned}
{\left[\begin{array}{c}
\dot{\hat{x}}^{\prime} \\
\hat{f}_{a}
\end{array}\right] } & =\left[\begin{array}{cc}
A+B K_{x} & 0 \\
0 & 0
\end{array}\right]\left[\begin{array}{c}
\hat{x} \\
\hat{f}_{a}
\end{array}\right]-\left[\begin{array}{c}
L_{x} \\
L_{f}
\end{array}\right](y-C \hat{x}) \\
& =\left[\begin{array}{cc}
\left(A+B K_{x}-L_{x} C\right) & 0 \\
-L_{f} C & 0
\end{array}\right]\left[\begin{array}{c}
\hat{x} \\
\hat{f}_{a}
\end{array}\right]+\left[\begin{array}{c}
L_{x} \\
L_{f}
\end{array}\right] y \\
& =[\underbrace{\left[\begin{array}{cc}
A+B K_{x} & 0 \\
0 & 0
\end{array}\right]}_{A_{x}}-\underbrace{\left[\begin{array}{c}
L_{x} \\
L_{f}
\end{array}\right]}_{L_{x}} \underbrace{\left[\begin{array}{cc}
C_{x} \\
0
\end{array}\right]}_{C_{x}}]\left[\begin{array}{c}
\hat{x} \\
\hat{f}_{a}
\end{array}\right]+\underbrace{\left[\begin{array}{c}
L_{x} \\
L_{f}
\end{array}\right] y}_{\tilde{x}}
\end{aligned}
$$

(6) can be re-written as:

$$
\dot{\widetilde{x}}=\left(A_{x}-L_{x} C_{x}\right) \tilde{x}+L_{x} y
$$

where $L_{x} \in R^{n \times p}$ and $L_{f} \in R^{m \times p}$ are the observer gains to be designed [See Proposition 1 and Theorem 1 below]. The feedback gain matrix $K_{x}$ is obtained by linear pole-placement design by the assumption that the fault effect in the control signal will be compensated, i.e. invoking the separation principle.

By solving the augmented state estimation system (7) the fault estimate $f_{a}$, is obtained as the lower compatible partition of $\tilde{x}$. Fig. (2) illustrates the partitioned structure according to Eqs. (1),(2) \& (7).

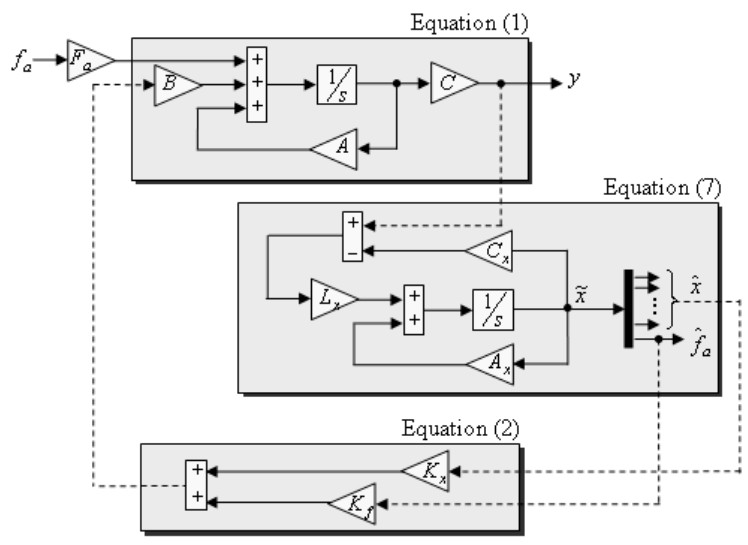

Fig. (2). ASO Fault estimation and compensation scheme.

\section{Proposition 1}

The estimation error system with fault corresponding to (1) and with the adaptive controller (2) and observer (4) is as follows:

$$
\begin{aligned}
\dot{x}-\dot{\hat{x}} & =A(x-\hat{x})-L_{x}(x-\hat{x})+B K_{f} \hat{f}_{a}+F_{a} f_{a} \\
\dot{e} & =\left(A-L_{x} C\right) e+B K_{f} \hat{f}_{a}+F_{a} f_{a} \\
{\left[\begin{array}{c}
\dot{e}_{x} \\
\dot{\hat{f}}_{a}
\end{array}\right] } & =\left[\begin{array}{cc}
A-L_{x} C & B K_{f} \\
L_{f} C & 0
\end{array}\right]\left[\begin{array}{c}
e_{x} \\
\hat{f}_{a}
\end{array}\right]-\left[\begin{array}{c}
F_{a} \\
0
\end{array}\right] f_{a}
\end{aligned}
$$

where $e_{x}=x-\hat{x} \in R^{n}$ is the state estimation error. The closed-loop system (4) can be viewed as an interconnected system consisting of a linear observer together with a nonlinear observer whose error dynamics contain an integral action which is basically the dual of integral control. The integral term $L_{f} C$ in (9) will force the estimation error $e_{x}$ to converge to zero, and hence compensate for the actuator fault $\left(f_{a}\right)$. Since the actuator fault $\left(f_{a}\right)$ is bounded, the compensation using integral action is achievable subject to a stability condition as discussed in Theorem 1 below.

Re-arranging (9) as:

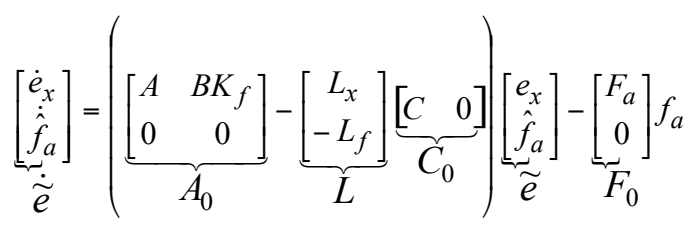


(10) can be re-written as:

$\dot{\tilde{e}}=\left(A_{0}-L C_{0}\right) \tilde{e}-F_{0} f_{a}$

The closed-loop system is thus described by (4) and (11). Since the actuator fault signals $\left(f_{a}\right)$ are bounded, one can always find a positive number $\beta$ such that $\beta>\left\|f_{a}\right\|$, where

$\|\cdot\|$ is Euclidean norm.

\section{Theorem 1}

Consider the closed-loop system described by (4) and (11) and assume that the pair $(A, B)$ is controllable and the pair $\left(C_{0}, A_{0}\right)$ is observable. If the observer gains $L$ in (11) are chosen such that there exists a s.p.d. matrix $P \in R^{(m+p) x(m+p)}$ satisfying:

$P\left(A_{0}-L C_{0}\right)+\left(A_{0}-L C_{0}\right)^{T} P=-\alpha \beta I$

where $\alpha>0$ and $\beta \geq\left\|f_{a}\right\|$, then $\tilde{e}=\left[\begin{array}{ll}e_{x}^{T} & \hat{f}_{a}^{T}\end{array}\right]^{T}$ will be contained in a bounded region around the equilibrium independent of initial conditions $e_{x}(0), \hat{x}(0)$ and $f_{a}(0)$. Furthermore, if the controller gains $K_{x}$ are chosen such that the matrix $A+B K_{x}$ is Hurwitz then $\hat{x}$ will also be contained in a bounded region around the equilibrium independent of $e_{x}(0), \hat{x}(0)$ and $f_{a}(0)$, meaning that the closed-loop system (4) and (11) is stable subject to the norm bound on $f_{a}(0)$, i.e. considering $f_{a}(0)$ as the "worst case" fault vector. This worst case follows as the appropriate region of stable attraction is defined in terms of $f_{a}(0)$ as defined below.

\section{Proof of Theorem 1}

Consider the following candidate Lyapunov function $V=\widetilde{e}^{T} P \widetilde{e}$ with its derivative along the trajectories of (11):

$\dot{V}=\widetilde{e}^{T}\left(P\left(A_{0}-L C_{0}\right)+\left(A_{0}-L C_{0}\right)^{T} P\right) \widetilde{e}-2 \widetilde{e}^{T} P F_{0} f_{a}$

Substitution of (12) and $\beta \geq\left\|f_{a}\right\|$ into (13) results in

$\dot{V} \leq-\beta\left(\alpha\|\widetilde{e}\|^{2}-2\left\|\widetilde{e}^{T} P F_{0}\right\|\right)$

By using the well known norm property [4]:
$\left\|\widetilde{e}^{T} P F_{0}\right\|=\sqrt{\widetilde{e}^{T} P F_{0} F_{0}^{T} P \widetilde{e}} \leq \sqrt{\lambda_{\max }\left(P F_{0} F_{0}^{T} P\right)}\|\widetilde{e}\|$

where $\lambda_{\max }(\cdot)$ denotes the largest eigenvalue of $(\cdot) . \delta$ can now be defined as:

$\delta=\frac{2}{\alpha} \sqrt{\lambda_{\max }\left(P F_{0} F_{0}^{T} P\right)}$

From (14) and (15) it follows that:

$\dot{V} \leq-\alpha \beta\|\tilde{e}\|(\|\widetilde{e}\|-\delta)$

A region of stable attraction can now be defined as $D_{\delta}=\{\tilde{e}:\|\tilde{e}\| \leq \delta\}$. Following (17) it can be concluded that $\dot{V}<0 \forall z \notin D_{\delta}$. Therefore, there exists $t_{0}>0$ such that $\tilde{e}(t) \in D_{\delta}, \quad \forall t>t_{0} \quad$ independent $\quad$ of $\quad e_{x}(0), \hat{x}(0)$ and $f_{a}(0)$, hence proving the first part of Theorem 1 .

Since the matrix $A+B K_{x}$ is Hurwitz, the subsystem (4) is a stable linear system subject to inputs $e_{x}$ that are bounded by $D_{\delta}$ around the origin. Therefore, $\hat{x}$ will also be bounded around the origin and hence the last part of Theorem 1 is proven.

Note that the standard linear system controllability and observability conditions guarantee the existence of the controller gain $K_{x}$ and observer gains $L$, satisfying the conditions in Theorem 1. The observer gain design criterion (12) can be solved using LMI computational methods, which are widely available. The size of steady state errors is defined by the region $D_{\delta}$, which can be decreased by enlarging the design parameter $\alpha$, as shown in (16). Theorem 1 is also applicable for linear systems subject to unknown bounded input disturbances/faults, i.e. $\beta$ is the bound of the input disturbances/faults.

\section{ACTUATOR FAULT COMPENSATION}

Once the FDI module indicates which actuator is faulty, the fault magnitude is estimated and a new control law is added to the nominal one to avoid the fault effect on the system. Moreover, here we only consider actuator faults only one fault is assumed to occur at the same time.

According to adaptive controller for actuator fault compensation in (2), here is re-written as: 
$u=\underbrace{K_{x}}_{u_{x}} \hat{x}+\underbrace{K_{f} \hat{f}_{a}}_{u_{a}}$

where $u_{x}$ is the control of the nominal system (fault-free case for which $K_{f}=0$ ), and $u_{a}$ is the compensating

control to be added to compensate for the actuator fault $\left(f_{a}\right)$ acting in the control channels. The FTC can therefore be achieved by replacing $u$ in (1) by (18) (see Fig. 2).

\section{FRICTION COMPENSATION CASE STUDY}

The control of systems that involve friction in moving mechanical components presents interesting challenges [11-13]. The tendency in recent years has been to go down a road of more and more detailed modelling of the friction phenomena in order to evoke an on-line friction compensation procedure, thereby attempting to cancel out the effect of the friction in the feedback loop [14].

FTC schemes for friction compensation can be developed which are adaptive in the sense of depending on bounded estimates of the friction forces. These requirements are satisfied when the friction force itself is considered as a fault effect. The friction force may be tolerable in the feedback system, allowing acceptable performance. However, if the system performance is degraded to a significant extent, exhibiting limit cycle oscillation, action needs to be taken either to remove the "faulty" component (e.g. replace it or giving lubricated bearing) or to invoke an automatic fault-tolerant strategy in the control system. It is reasonable to consider the friction force as a fault as the friction in a mechanical system is an unwanted phenomenon in the majority of real systems [10].

This is a natural development of the modelling requirements in robust and non-linear control and estimation. Despite several important studies the friction modelling problem remains a very difficult challenge, mainly because of the uncertain dynamic characteristics involved and that friction characteristics change over time due to, for example to wear, temperature and humidity [14]. From a control point of view, friction compensation strategies that require a detailed model of the friction characteristics have limitations arising from non-smooth non-linearity and the fact that friction modelling remains an imprecise subject, thereby resulting in a robustness problem.

The friction forces acting in a mechanical system can be viewed as a specific type of actuator fault signals which act on the $m$ control channels, whilst depending on linear velocity components (viz. as the friction forces are dependent on velocity). Hence, this is a special case of actuator fault compensation which has wide practical use in many mechanical and mechatronic systems. The practical value becomes clear from the example below, as the stability conditions are strong. Furthermore, the friction force bounds should be considered no greater than their static friction values which are often known a priori from bearing manufacturer specifications $[11,12]$.
The example below shows that methods of fault estimation allied to FDI theory [13] can be used to obviate the use of very complex friction modeling, so popular currently in the control literature. Friction force estimates can be used within an FTC structure to provide on-line friction compensation. The friction estimates provide important robustness indicators for friction compensator design.

Following (1), the system subject to friction forces acting in up to $m$ of the input channels independently (e.g. on some or all of the actuated joints of a robotic system) can be re-written as:

$$
\begin{aligned}
& \dot{x}=A x+B\left[u-f_{\text {fric }}\right] \\
& y=C x
\end{aligned}
$$

where $f_{\text {fric }}=\left[f_{\text {fric }}^{1}, \ldots, f_{\text {fric }}^{m}\right]^{T}$ represents the friction forces acting on the system. Hence, the nonlinear friction forces that reduce the effective control forces according to (19)\} for given control inputs, can be represented as actuator faults. The proposed friction compensation methods do not require a model of the nonlinear friction forces $\left(f_{\text {fric }}\right)$. The method only requires that the friction forces should be bounded, which is a valid assumption as discussed above.

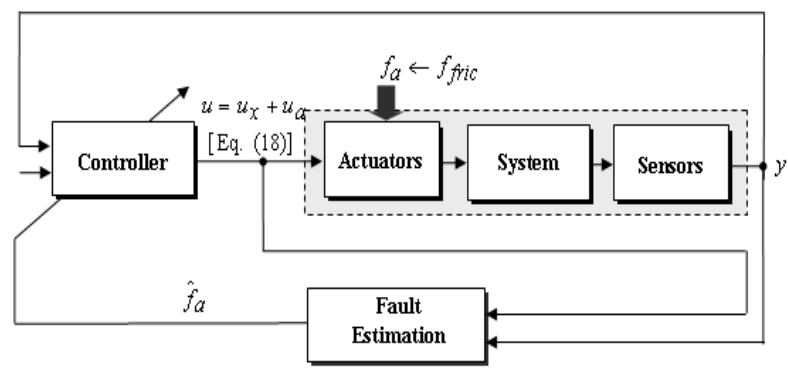

Fig. (3). A Fault-tolerant control view to friction compensation.

Inverted Pendulum Example: To illustrate the above discussion a tutorial example of the friction compensation problem is considered using a non-linear simulation of the inverted pendulum and cart together with simulated Stribeck friction force. The friction compensation involves estimation of a scalar friction force acting against the scalar control force $u(t)$, as illustrated in (Figs. 3 \& 4). The cart is linked by a transmission belt to a drive wheel which is driven by a DC motor to rotate the pendulum into vertical position in the vertical plane by force control on the cart. The equations of motion including friction on the cart are

$$
\begin{aligned}
& (M+m) \ddot{x}_{p}+F_{x} \dot{x}_{p}+m\left(\ddot{\theta} \cos \theta-\theta^{2} \sin \theta\right)=u(t)-f_{\text {fric }}\left(\dot{x}_{p}\right), \\
& J \ddot{\theta}+F_{\theta} \dot{\theta}-m l g \sin \theta+m l \ddot{x}_{p} \cos \theta=0
\end{aligned}
$$


where $x_{p}, \theta$ are the cart position and the pendulum angle, respectively. The particular values of the system parameters such as rod length and masses, etc, are given in Table 1 of [3].

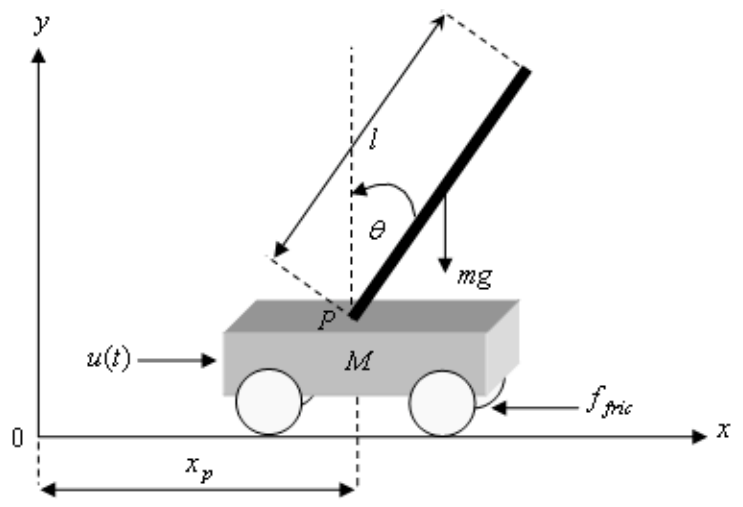

Fig. (4). Inverted pendulum system.

For simulation purposes the friction force acting on the cart is described by the discontinuous Stribeck friction model [18]:

$f_{\text {fric }}=g\left(x_{p}\right) \operatorname{Sign}\left(x_{p}\right)$,

where $\operatorname{Sign}\left(x_{p}\right) \in\left\{\begin{array}{ccc}\{-1\} & \text { if } & x_{p}<0 \\ {[-1,1]} & \text { if } & x_{p}=0 \\ \{1\} & \text { if } & x_{p}>0\end{array}\right.$

$g\left(x_{p}\right)=F_{c}+\left(F_{s}-F_{c}\right) \exp \left(-\left|x_{p}\right| / v_{s}\right)^{\delta} \quad$ is the Stribeck friction function with $F_{c}$ and $F_{S}$ are the Coulomb and static friction levels, respectively and $v_{S}, \delta>0$ are the Stribeck velocity and shaping parameters, respectively. In the simulation the following parameter values are used:

$$
F_{c}=25 N, F_{s}=30 N, v_{s}=0.15 \mathrm{~ms}^{-1} \text { and } \delta=2
$$

A linearization of the left hand side of (21) results in the perturbation states $x=\left[x_{p}, \dot{x}_{p}, \theta, \dot{\theta}\right]$ corresponding to the equilibrium point: $x_{p}=\dot{x}_{p}=\dot{\theta}=\theta=0$. The linearised system triples corresponding to the single input $u(t)$ and measurements $y \in \Re^{3}$. The three measurements (cart position, pendulum angular position and cart velocity) replicate the measurements of the laboratory system.

$$
\begin{gathered}
A=\left[\begin{array}{cccc}
0 & 0 & 1 & 0 \\
0 & 0 & 0 & 1 \\
0 & -1.9333 & -1.9872 & 0.0091 \\
0 & 36.9771 & 6.2589 & -0.1738
\end{array}\right], B=\left[\begin{array}{c}
0 \\
0 \\
0.3205 \\
-1.0095
\end{array}\right] \\
\text { and } C=\left[\begin{array}{llll}
1 & 0 & 0 & 0 \\
0 & 1 & 0 & 0 \\
0 & 0 & 1 & 0
\end{array}\right]
\end{gathered}
$$

In this study, it is assumed that only the position measurement of the cart $\left(x_{p}\right)$ and the angle of the pendulum $(\theta)$ are available for the feedback loop, i.e. $C=\left[\begin{array}{llll}1 & 0 & 0 & 0 \\ 0 & 1 & 0 & 0\end{array}\right]$. The friction compensation gain is chosen as $K_{f}=1$, corresponding to the bound arising from the static friction force $F_{s}=30 \mathrm{~N}$ \{i.e. $\left.\beta<F_{s}=30\right\}$. It can be verified that the augmented system pair $\left(C_{0}, A_{0}\right)$ as in (11) is observable. The observer gains $L=\operatorname{col}\left(L_{x}, L_{f}\right)$ are designed such that the eigenvalues of $\left(A_{0}-L C_{0}\right)$ are placed at $-1200,-1500$, $-1800,-2100$ and -3000 satisfying condition (12), is given by:

$$
L=\left[\begin{array}{cc}
0.0380 & -0.0048 \\
-0.0038 & 0.0518 \\
0.3792 & -0.1690 \\
-0.1297 & -0.8841 \\
--3.3432 & 0.8941
\end{array}\right] \times 1.0 \mathrm{e}^{3}
$$

The controller gain $K_{x}$ is designed by placing the eigenvalues of $\left(A+B K_{x}\right)$ at $-4.5,-6.0,-7.5$ and -9.0. Simulation results for given initial values: $x(0)=\left[\begin{array}{llll}1.1 & -1.1 & 0 & 0\end{array}\right]^{T}$ and $f_{a}(0)=1$ are shown in (Figs. 5 and $\left.\mathbf{6}\right)$.
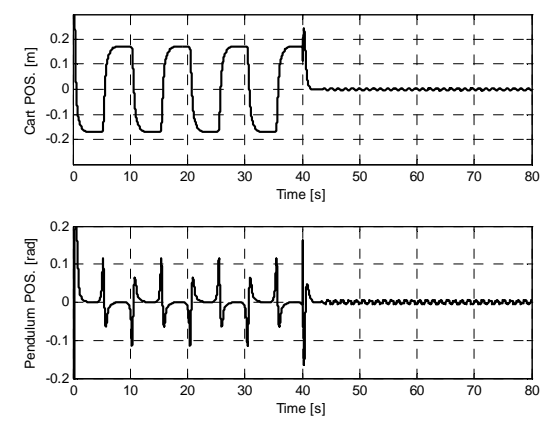

Fig. (5). ASO friction compensation results for the friction compensation activated at $t=40 \mathrm{~s}$. 
Fig. (5) shows that for $t<40 s$ the augmented state $\hat{f}_{\text {fric }}$ is switched off, illustrating that the inverted pendulum model system exhibits limit cycle oscillation around the vertical equilibrium point (the origin). This is because the cart, which is affected by the friction, exhibits stick-slip motion. For $t>40 s$ the compensator is activated via $K_{f} f_{a}$ in (18) so

that the limit cycle oscillation is significantly reduced to very a small neighbourhood around the equilibrium point as predicted by Theorem 1. After compensation the amplitude of the pendulum angular oscillation is less than $2 \mathrm{mrad}$ and the amplitude of the cart stick-slip motion is less than $1 \mathrm{~mm}$.
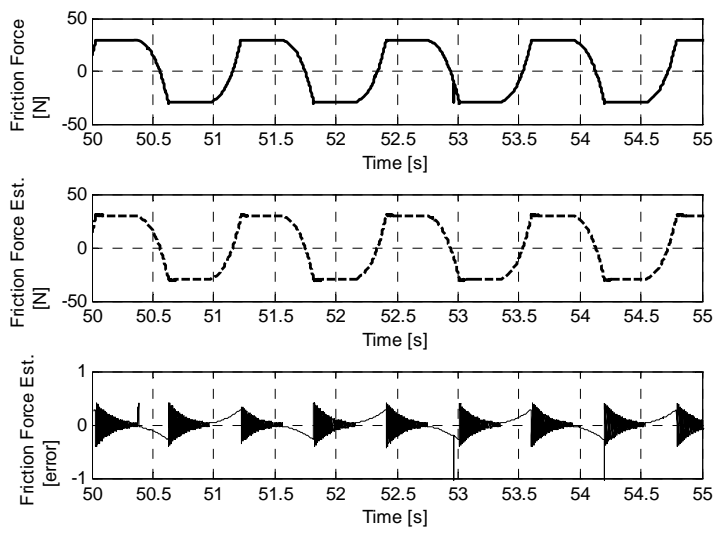

Fig. (6). Comparison of friction force $\left(f_{\text {fric }}\right)$ and its estimate

$\left(\hat{f}_{\text {fric }}\right)$.

These results can be achieved since the friction force is accurately estimated by the compensation term $K_{f} f_{a}$ as depicted in Fig. (6). Some spikes occur in the friction estimation/compensation error as shown in the lower part of Fig. (6) which are due to the discontinuous nature of the friction force during the transition of the stick-slip motion. This discontinuous nature of the friction cannot be followed immediately by the friction estimate.

\section{CONCLUSION}

This paper proposes a new strategy for FTC making use of for fault estimation and compensation via the design of an augmented state observer (ASO) whose integrated estimation error has the effect of compensating each fault signal. The integral of the observer estimation error is represented according to an augmented system description. In this paper the additional states are shown to be estimates of actuator faults.

An adaptive control fault compensation method has been developed and analyzed and the new compensating control is computed using the estimation information, derived from bounds on the faults. A tutorial example is given of the compensation of friction force in the inverted pendulum, as an FTC problem considering the friction force as an input channel (actuator) fault.

It is clear from this study that the friction compensation problem for a mechanical system can be viewed as an FTC problem which does not require a model of the friction forces. It is interesting to note that although most studies consider the friction force to have an uncertain effect on the system it is more constructive here to view the friction force as a particular fault. From a practical standpoint this method can be implemented well on real-time application systems.

Additionally, when compared with the model-based compensation methods the advantage gained is that the model-robustness problem is obviated and this is considered of practical significance. For the friction problem, the on-line fault estimation/compensation strategy proposed circumvents the complexity problem that can arise when model-based friction compensation methods are used. As friction phenomena are so difficult to model, the friction estimation approaches may yield better robustness and improved friction compensation.

The combined fault estimation and compensation problem provides a powerful method of loop-transfer recovery, enabling the Separation Principle to be reached as the faults and/or uncertainties are estimated. Whilst the example given is an illustration based on the friction compensation problem, the theory and approach has wide application to more complex problems in which actuator, sensor faults as well as multiplicative faults and unknown input signals can all be compensated together using the system description and stability conditions of Section II. A discussion of this will be the subject of an extended study.

\section{ACKNOWLEDGMENT}

S. Klinkhieo acknowledges $\mathrm{PhD}$ scholarship funding from the Synchrotron Light Research Institute (SLRI) under the Royal Thailand Government.

\section{NOMENCLATURE}

$\begin{array}{lll}\text { FTC } & = & \text { Fault-Tolerant Control } \\ \text { FDI } & = & \text { Fault Detection and Isolation } \\ \text { ASO } & = & \text { Augmented State Observer } \\ \text { LPV } & = & \text { Linear Parameter Varying } \\ \text { LMI } & = & \text { Linear Matrix Inequality }\end{array}$

\section{REFERENCES}

[1] J. Chen and R.J. Patton, "Standard Hळ filtering formulation of robust fault detection", IFAC Symposium SAFEPROCESS 2000, Budapest, pp. 256-261, June 14-16, 2000.

[2] J. Bokor and G. Balas, "Detection filter design for LPV systems - a geometric approach,” Automatica, vol. 40, pp. 511-518, 2004 
[3] C. Edwards and S.K. Spurgeon, Sliding Mode Control: Theory and Applications, Taylor and Francis, London, 1998.

[4] C. Edwards, S.K. Spurgeon and R.J. Patton, "Sliding mode observers for fault detection and isolation", Automatica, vol. 36, pp. 541-553, 2000.

[5] C. DePersis and A. Isidori, "A geometric approach to nonlinear fault detection and isolation", IEEE Transactions on Automatic Control, vol. AC-46(6), pp. 853-865, 2001.

[6] R.J. Patton, "Fault-tolerant control: The 1997 situation (survey)", IFAC SAFEPROCESS'97, Hull, UK, August 26-28, 1997. vol. 2, pp. 1033-1055.

[7] M. Blanke, M. Kinnaert and J. Lunze \& Staroswiecki M, Diagnosis and Fault-Tolerant Control, Springer-Verlag, 2003.

[8] M. Blanke, C. Frei, F. Kraus, R. J. Patton and M. Staroswiecki. "What is Fault-tolerant control?" Proc. IFAC Sympo. On Fault Detection Supervision and Safety for Technical Processes, Budapest, 2000, vol. 1, pp. 40-51.

[9] J. Chen and R. J. Patton, Robust Model Based Fault Diagnosis for Dynamic Systems, Kluwer Academic Publishers 1999.

[10] R. J. Patton, S. Klinkhieo and D. Putra, "A fault-tolerant control approach to friction compensation", Invited Session Paper, European Conference on Control 2009 (ECC'09), August 23-26, Budapest, Hungary, 2009.

[11] B. Armstrong-Hélouvry B, P. Dupont, and W. C. Canudas de, "A survey of models, analysis tools, and compensation methods for the control of machines with friction", Automatica, vol. 30, no.7, pp. 1083-1138, 1994.

[12] H. Olsson, K.J., Aström, W.G. Canudas de, M. Gäfvert and M. Lischinsky, "Friction models and friction compensation", European Journal of Control, vol. 4, pp. 176-195, 1998.

[13] B.S.R. Armstrong and Q. Chen, "The Z-properties chart", IEEE Control Systems Magazine, vol. 28, no. 5, pp. 79-90, 2008.

[14] B. Bona and M. Indri, "Friction Compensation in Robotics: an Overview", Proc. the joint 44th IEEE Conference on Decision and Control and the European Control Conference, 2005, pp. 4360-4367.

[15] F.J. Uppal and R.J. Patton, "Neuro-fuzzy uncertainty de-coupling: A multiple-model paradigm for fault detection and isolation", Int. Journal of Adaptive Control \& Signal Processing, (Invited Special Issue Paper), vol. 19, no. 4, pp. 281-304, 2005.

[16] R. J. Patton and J. Chen, "Robust fault detection of jet engine sensor systems using eigenstructure assignment", Journal of Guidance, Control \& Dynamics, vol. 15, no. 6, 1491-1497, 1992.

[17] J. Chen and R. J. Patton, "Optimal filtering and robust fault diagnosis of stochastic systems with unknown disturbances", IEE Proceedings on Control Theory \& Applications, vol. 143 no. 1, pp. 31-36, 1996.

[18] D. Putra, L. Moreau and H. Nijmeijer, "Observer-Based Compensation of Discontinuous Friction", Proceedings of the $43^{\text {rd }}$ IEEE Conference on Decision and Control, the Bahamas, 2005, pp. $4940-4945$.

(C) Patton and Klinkhieo; Licensee Bentham Open.

This is an open access article licensed under the terms of the Creative Commons Attribution Non-Commercial License (http://creativecommons.org/licenses/by-nc/3.0/) which permits unrestricted, non-commercial use, distribution and reproduction in any medium, provided the work is properly cited. 